Revue d'histoire de l'Amérique française

ARS REVUE D.HISTOIRE DE L'AMÉRIQUE FRANÇAISE

\title{
Le journal d'un militaire au Nord-Ouest canadien (suite et fin)
}

\section{George F. G. Stanley}

Volume 10, numéro 3, décembre 1956

URI : https://id.erudit.org/iderudit/301776ar

DOI : https://doi.org/10.7202/301776ar

Aller au sommaire du numéro

Éditeur(s)

Institut d'histoire de l'Amérique française

ISSN

0035-2357 (imprimé)

1492-1383 (numérique)

Découvrir la revue

Citer ce document

Stanley, G. F. G. (1956). Le journal d'un militaire au Nord-Ouest canadien (suite et fin). Revue d'histoire de l'Amérique française, 10(3), 416-430.

https://doi.org/10.7202/301776ar d'utilisation que vous pouvez consulter en ligne.

https://apropos.erudit.org/fr/usagers/politique-dutilisation/ 


\title{
DOCUMENTS INÉDITS
}

\author{
LE JOURNAL D'UN MILITAIRE AU NORD.OUEST CANADIEN * \\ (Suite)
}

Mercredi, 13 mai. Le matin on alla tirer à la cible, à une distance de 300 verges. Le reste de la journée se passa comme d'habitude. Alain et Jolicœur revenant de monter la garde ont eu chacun un méchant mal de gorge. Dans la nuit de mercredi en venant au jeudi je fus très malade. Je voulais aller communier pour l'Ascension le lendemain.

Jeudi. Temps nuageux, vent, pluie et grêle. Grande messe à $10 \mathrm{hrs}$. à la mission du Père Lacombe. Réellement le sort est contre moi; on me nomme pour la garde. Je ne peux décrire les souffrances que j'endurai pendant cette nuit épouvantable. Pour comble de malheurs, le café qu'on avait coutume de nous donner à minuit n'arriva pas. Puis le mal de dents que j'avais depuis hier augmenta, car le vent soufflait très fort; tellement que plusieurs tentes furent jetés à terre par le vent. Je ne pouvais m'arrêter 2 minutes sans me voir les pieds engourdis par le froid. Je compare cette nuit à celle passée sur les chars plates formes.

Le lendemain, vendredi, les rations nous furent distribuées qu'à 9 hrs. Le capt. Pinault et le lieutenant ${ }^{28}$ vinrent me trouver le lendemain matin sur mon poste et me demandèrent comment j'avais passé ma nuit de garde. Cela me rendit quelque peu le courage, car j'écrasais de fatigue. Ce matin à $10 \mathrm{hrs}$. Jolicœur et Alain sont entrés à l'ambulance, cependant ils ne sont pas beaucoup malades, heureusement. Relève de garde à $10 \mathrm{hrs}$. Je ne dormis pas de la matinée. Je passai mon temps à écrire des lettres. La parade se fit dans les casernes. L'après-midi je dors jusqu'à 5 hrs. Puis j'écris 3 lettres. Chose extraordinaire aujourd'hui, on eut de la viande rotie dans la graisse de lard ! On apprend que 15 teams ont été pillés par les sauvages et que les conducteurs sont prisonniers. Riel et Gabriel Dumont se sont enfuis. La plupart des Métis se rendent à Middleton. Les sau-

* Voir la Revue, vol. X, no 2: 263-278.

28 Capitaine L. F. Pinault, commandant de la compagnie no 7 ; Lieutenant Pantaléon Pelletier. Lieutenant Jean-Charles Routhier de la 7 ième compagnie avait accompagné le détachement qui a quitté Calgary le 10 mai. 
vages seuls restent à combattre. ${ }^{29}$ Nous nous attendons de nous frotter lundi ou mardi. ${ }^{30}$ Vendredi soir nous avions eu une nouvelle alerte. A $10 \mathrm{hrs}$. du soir on vint demander 10 hommes. Je fus assez heureux pour en faire partie avec DeGuise et Paradis. ${ }^{31}$ On distribue des cartouches, puis nous marchons vers la ville, car les métis se battaient, disait-on, avec 3 ou 4 de nos soldats, mais on rencontra le premier piquet de sergent et tout était fini.

Samedi, 16. Parade de 5.30. Je m'en exempte et aussi de celle de $10 \mathrm{hrs}$. L'après-midi nous avons un tir de $400 \mathrm{vgs}$. Je fais 7 points. Dans la soirée de samedi le sergent-major ${ }^{32}$ vint nous avertir de se coucher tout habillé et il avait un corps de cartouches à notre disposition. Les gardes furent triplés le soir et grâce à cette garde nous n'eûmes pas d'alerte. Cependant ils firent sortir la garde au moins 20 fois dans la nuit pour chasser quelques rodeurs sauvages des alentours du camp. Je dormis très bien toute la nuit. Les sauvages sont partis de Calgary aujourd'hui pour leurs réserves ou ils le feignent, et il en arriva 150 autres sauvages Pieds-Noirs à 2 milles du camp.

Dimanche, 17 mai. Ce matin je me lève à $5 \mathrm{hrs}$. Je vais à la confesse et communie à la mission. Ce fut le Père Claude qui entendit ma confession. La mission se trouve à une distance d'un mille et demie du camp. A $10 \mathrm{hrs}$. je retourne à la grande messe avec le bataillon. Il fait une chaleur écrasante. Nous avons un rose Beef au diner! L'après-midi nous partons pour aller louer des chevaux de selle afin de se rendre aux Montagnes Rocheuses, mais on ne put en trouver, je revins au camp et je me couche. A 3.30 hrs. P.M. le vent et la pluie recommencent. Je plains la garde! Le soir après le souper il fait beau. Je pars avec Lavoie, DeGuise et Poudrier ${ }^{33}$ pour aller voir nos amis les sauvages et en vue de trafiquer. Nous faisons 10 milles dans la plaine, puis de retour au camp on m'apprend que je suis de garde,

${ }^{29}$ Le 12 mai, les miliciens du général Middleton avaient écrasé les métis et capturé le village de Batoche, quartier-général de Riel. Riel se constitua prisonnier le 15 mai. Gabriel Dumont put gagner les Etats-Unis. Faiseur d'enclos (Poundmaker) se livra à Middleton le 26 mai. Le 28 mai le général Strange vainquit les Cris de Gros Ours au Butte-aux-français. Le 2 juillet, Gros Ours se livra à la Police Montée au Fort Carlton.

${ }_{30} \mathrm{La}$ défaite des Métis et la prise de Riel mettaient fin à la campagne militaire. Les troupes anticipaient leur retour à Québec. compagnie.

31 Charles DeGuise, soldat, $7^{\mathrm{e}}$ compagnie; Jules Paradis, caporal, $7^{\mathrm{e}}$

32 Edmond Trudel, sergent-major, $7^{\mathrm{e}}$ compagnie. compagnie.

33 Arthur Lavoie, soldat, $7^{e}$ compagnie; Alcide Poudrier, caporal, $7^{e}$ 
car la garde était de 28 hommes ce jour-là. On redoutait une attaque. Nous eûmes un assez beau temps la nuit, mais il faisait très noir. Le soir à la garde, je reçois une lettre d'Eva.

Lundi. Je me couche à $5 \mathrm{hrs}$. du matin, puis je dine et déjeune à $11 \mathrm{hrs}$. Je réponds à Eva. L'après-midi encan des effets du quartier-maître. Il trouva moyen encore de nous piller dans cet encan. Elle doit continuer demain où après demain. Le reste de la journée se passa comme d'habitude.

Mardi, 18 mai. Lever à $7 \mathrm{hrs}$. Plus de parade. Nous ne faisons que la garde tous les 2 jours. Je suis de garde d'aujourd'hui. Pendant toute la nuit on entendit chanter les sauvages. Ils commencèrent leurs dances et leurs chants à $101 / 2$ jusqu'à 5 hrs. du matin.

Mercredi, 19 mai. Même routine. Le soir le caporal Poudrier, l'adjutant Casgrain, Tanguay, Dorion, Chabot ${ }^{34}$ arrivent. Ils nous apprennent que tous les sauvages ont quitté leurs réserves et ils s'avancent contre les troupes. On espère partir bientôt. Ce soir je veillai jusqu'à minuit en ayant le soin de cacher la lumière, et j'écrivis deux lettres, une à $\mathrm{E}$. et l'autre à M. Lazare Noël. Cette nuit encore on s'attendait à une attaque.

Jeudi. Temps pluvieux. C'est très ennuyant. Aujourd'hui je suis malade. Cependant je suis obligé de faire la garde encore, le docteur cependant m'exempta. Ce soir-là on attendait les sauvages; baionnettes et cartouches au bout du fusil.

Vendredi, 22 mai. Arrivée de ceux qui avaient été reconduire les munitions. Beau et mauvais temps. On reçoit de mauvaises nouvelles. Tous les sauvages ont quitté leurs réserves et s'avancent du côté des blancs! On parle de nous renvoyer à Québec que dans 6 mois au plus tôt. On doit nous envoyer en avant à Edmunton d'abord puis sur le théâtre de la guerre. Hourrahs pour nous !

Samedi. Grand fatigue party. Je me lève avec un grand mal de gorge. Je vais voir le docteur de l'hôpital pour me donner des remèdes. Pendant toute la journée je fus très malade. Je réussis à me faire exempter de tout devoir jusqu'à parfaite guérison. On reçoit de bonnes nouvelles aujourd'hui. Le soir on danse des quadrilles dans notre chambre, car notre cook possède un violin.

Dimanche, 24 mai. Lever à 5.30 puis nous allons à $10 \mathrm{hrs}$. entendre la grande messe à la mission. Sermon du Père Lacombe en anglais, en français, puis en cris. L'après-midi je reçois une

34 Capitaine Philippe du Perron Casgrain; Narcisse Tanguay, Noël Dorion, soldats, $7^{\mathrm{e}}$ compagnie; Joseph-E. Chabot, sergent, $7^{\mathrm{e}}$ compagnie. 
lettre de Clara avec un portrait. La journée se passa comme d'habitude. Je me couchai très malade.

Lundi, 25 mai. Nous nous préparons à fêter la fête de la reine avec éclat. A $111 / 2 \mathrm{hrs}$. grande parade, nous réussissons très bien. 300 cartouches blanches furent tirées par nous. Puis l'après-midi il y eu des courses de toutes espèces les yeux bandés et les pieds dans une poche. 18 hommes du bataillon et 18 de Calgary tirent sur la corde un tug of war. Pour la fin les Pieds-Noirs nous donnions une danse de guerre. A $8 \mathrm{hrs}$. bal des officiers, la valse et le polka étaient l'ordre du jour. Ils ne dansèrent que 2 ou 3 autres danses. Pendant ce temps, les soldats dansaient entre eux dans leurs quartiers. A $4 \mathrm{hrs}$. départ des officiers, alors nous envahissons les salles, puis nous dansons et ensuite le réveillons. Pendant le bal le quartier-maître ${ }^{35}$ se fit voler une camisole, une paire de caleçons puis 2 serviettes ...

Mardi. Temps pluvieux et malsain. Je ne prends que le souper à $8 \mathrm{hrs.,}$ tant j'étais abruti. Je m'ennuyai beaucoup. J'acheta une petite pipe à un sauvage Pied Noir.

Mercredi. Lever à huit heures, temps assez beau. On va tirer à la cible à 400 verges. Je fais 8 points. Diner, puis nous attendons les lettres avec impatience. Je reçois 4 lettres, une de François, Plidor, Maman et Eva. Le soir je veille très tard pour répondre à ces lettres.

Jeudi. Pas de changement dans notre situation, seulement que le départ est proche. Mais on ne peut encore préciser. Pendant la nuit de jeudi à vendredi nous fîmes une razzia dans le store du quartier-maître. On récolta au moins $50 \mathrm{lbs}$ de cassonade.

Vendredi, 29 mai. L'avant-midi se passa comme d'habitude. Puis l'après-midi nous avons un tir à la cible de 10 rondes. Je fis un boulseye. Le soir après le souper j'allai voire les sauvages. Je visitai toutes les cabanes de ceux-ci et nous achetâmes une pipe.

Samedi. L'après-midi nous lavons encore nos casernes.

(L'auteur n'a rien inscrit à son journal du 30 mai au 24 juin).

24 juin. Réveil à $6 \mathrm{hrs}$. Aussitôt le réveil sonné, nous qui avions coutume de flâner dans nos lits une demi-heure après, nous nous mîmes tous à chanter ' $O$ Canada'. Puis à 7 hrs. nous allâmes à la porte des casernes tirer chacun 8 cartouches blanches, et cela de nous-mêmes. Il se trouvait quelques soldats du

35 Capitaine Aimé Talbot, quartier-maître. 
No. 6 parmi nous. Ensuite nous hissons sur nos casernes le pavillon français avec des hourrahs et des discours patriotiques. Malgré les ordres des officiers de cesser, nous continuons. A $91 / 2$ nous avons une messe et un discours à la mission. Après la messe on nous avertit et la garde est averti de nous arrêter si nous voulions sortir, car le Colonel ne voulait pas nous laisser fêter notre fête nationale sous des prétextes qui ne sont pas dignes d'un Canadien. Cependant toute la compagnie, nous nous sommes sauvés en ville et là nous nous sommes amusés toute la journée; même nous avons dépensé $\$ 4$ par - et revenir au camp à $10 \mathrm{hrs}$. Là nous nous sommes couchés car - nous passons pour des hommes braves et nous recevons des félicitations de tous.

Jeudi, 25 juin. Drill à $7 \mathrm{hrs}$. Puis on attend l'enquête qui devait avoir lieu à $11 \mathrm{hrs}$. Mais cette enquête fut envoyée aux Calendes Grecques. Après le dîner, le Capt. Pinault, Lieut. Pelletier, les Cols. Amyot et Evanturel sont partis pour MacLeod. Pas de parade l'après-midi. Hier, la comp. No. 10 est arrivée à temps pour fêter la St. Jean Baptiste. Le soir nous devions aller voir la tribu des Sarcies ${ }^{36}$ à 10 milles d'ici, car ils ont commencé leurs danses du soleil; mais la partie est remise au lendemain.

26 juin Vendredi. Le matin il y eut parade avant le déjeuner, mais par une permission du Capt. Pennée ${ }^{37}$ nous partîmes, 6 soldats, le matin à $8 \mathrm{hrs}$. pour rendre à la réserve des Sarcies. A pied la distance est de 22 milles aller et retour. Nous fîmes le trajet, aller, en $21 / 2 \mathrm{hrs}$. A un mille de la réserve nous prîmes notre dîner dans un petit bois et nous trouvâmes des fraises. Après s'être reposé une heure on se rend à la réserve. Cependant on fit obligé d'attendre car la danse ne devait commencer que 2 hrs. plus tard, car ils attendaient d'autres sauvages qui devaient y prendre part. Je profitai de ce temps pour faire l'inspection de la réserve. Ils ont un magnifique terrain près de la rivière Elbow. Toutes les tentes ou Tipi forment un grand cercle; au milieu de celui-ci était dressé une espèce de Forum en feuillage, au centre duquel était dressé le poteau de guerre ou de torture. Cette réserve compte 75 familles. Pendant que je visitais le camp je fus rencontré d'un français, un pur Parisien, le Comte de Montjean, qui était au Nord-Ouest depuis le mois comme promeneur. Je passai la journée avec lui.

36 Les Sarcis constituent une tribu indienne assez petite. Bien qu'ils fassent partie de la confédération Pied Noir, ils parlent la langue Athabascaine. Leur culture est celle des sauvages des prairies. Ils occupent aujourd'hui une réserve tout près de Calgary.

37 Capitaine Frank Pennée, commandant de la compagnie no 5. 
A une heure commença leurs danses. D'abord les sorciers arrivent et se mettent dans une place réservée; on disait qu'ils n'avaient pas mangé depuis 15 jours et ils en avaient tout l'air. Ensuite tous les sauvages se rangèrent alentour du forum, la plupart ayant leurs squaws derrière eux. On apporta alors un grande nombre de chaudrons dans lesquels se trouvaient du riz ou du thé. Après avoir engloutis toutes ces provisions, ils commencèrent leurs danses avec acclamations des enfants ou papouses et des squaws. Après avoir dansé une heure ou à peu près, ils firent venir le supplicié, celui de devait être reçu brave. Il était presque nu. On le fit coucher et alors commencèrent les tortures. D'abord on le perça l'estomac avec des lances. On lui coupe deux lanières de peaux dans lesquels on passe deux bois; puis à ces bois on met les cordes. Il résista à cette torture sans broncher et sans manifester quant à l'extérieur le moindre signe de souffrance. Attaché ainsi il se laisse pendre aux cordes ce qui lui doit faire un mal horrible et on ne laisse aller seulement que quand les chairs se brisent. Alors il se lève et par le fait même il est reçu brave. Après ces actes de barbaries ils continuent leurs danses simulant des combats, ils scalpent, enlèvent des cheveux, etc. Celui qui est reçu brave a un cheval de la nation; sur ce cheval on dessine des figures lesquelles je me fis expliquer. Chaque figure semblable indique soit qu'il ait tué ses ennemis, volé de chevaux, ou le nombre de fois qu'il avait été à la guerre. Celui-ci avait volé 12 chevaux, tué 15 hommes, était allé 10 fois à la guerre etc., avait remporté un très grand [nombre de] chevelures etc.

Enfin nous quittâmes leur réserve à $71 / 2$ du soir. A peine étions-nous partis que le temps se prépara au mauvais, et il tomba beaucoup de grêle. J'en ramassai de très gros morceaux. Le plus comique de l'affaire c'est qu'avec deux de mes compagnons je rencontrai 4 sauvages à cheval. Sur leur invitation de monter en croupe, nous nous laissâmes persuader et ils nous galopèrent ainsi environ 8 milles. Nous traversèrent la rivière Elbow à gué, etc. Lorsque je débarquai je ne pouvais marcher. Nous leurs offrons chacun $25 \mathrm{cts}$, mais ils refusent, nous demandant [illisible] une piastre. Alors nous les envoyons au... (sic) mais ils nous suivent jusqu'aux casernes et vont se plaindre au commandant qui les envoie paître. Ils revinrent 3 jours de suite nous demander leur piastre. Nous fûmes obligés de les mettre forcément à la porte du fort. J'arrive au camp très harassé et cette nuit-là je dormis très bien.

27 juin, samedi. Parade du matin, il n'y avait personne de notre compagnie sur le champ. Cependant, malgré que je ne fus 
pas capable de me remuer les jambes, on me fit driller une heure et demie sous peine d'aller à la garde. Après le déjeuner j'écris à Papa et Adolphe. L'après-midi on reçoit des télégrammes que nous promettent un prompt retour. Le reste de la journée se passe comme d'habitude.

Dimanche. Parade à $10 \mathrm{hrs.} \mathrm{Puis} \mathrm{nous} \mathrm{nous} \mathrm{rendrons} \mathrm{à} \mathrm{la}$ mission pour la messe. Après la messe, inspection de casernes. L'après-midi nous n'avions d'autres choses à faire que de la passer coucher, car il faisait beau, mais très chaud. La malle arrive et chacun lit ses lettres et tout retombe dans un silence occasionné par l'ennui, etc.

Lundi, 29 juin. Fêtes de St. Pierre et St. Paul, Grand congé, pas de parade ni aucun devoir. Le matin nous avons une messe à la mission spécialement pour nous à laquelle le Rév. Père Lacombe ajouta un petit sermon qui n'était autre que ses adieux. Il était très attristé de notre prochain départ.

Mardi, 30 juin. Parade à 7 hrs. Je m'exempte de cette drill encore prétendant une maladie quelconque; puis, etc., ${ }^{38}$

Mercredi, 1 juillet. Dominion Day. Tout Calgary s'était donné la main pour célébrer cette fête avec pompe. Il y eut toutes espèces de jeux pendant toute la journée. Le matin on commença par le tire à cible; puis l'après-midi vinrent les courses et les autres amusements qui forment le Sport des Anglais. Je reçus 2 lettres ce jour-là. Il me fallait cela pour m'enlever un peu d'ennui. Nos gens qui étaient à MacLeod nous étaient revenus mardi nous disant que les gens de MacLeod devaient partir de cette place jeudi au matin, 2 juillet.

Jeudi, 2. Même routine. L'après-midi vers les $5 \mathrm{hrs.} \mathrm{nous}$ apercevons sur une colline à un mille du camp, une longue file rouge qui n'était autre que nos frères de MacLeod. Alors nous allâmes tous à leur rencontre pour leur souhaiter le bienvenu. Il n'y a rien plus touchant que cette scène famille (sic) pour ainsi dire. Que de poignées de main, que de questions ! Enfin nous les amenons souper avec nous en nous privant toutefois de nos rations, mais que nous importait, le bataillon commencent à se reformer de nouveau et cela un signe précurseur du départ. Nous passâmes une agréable soirée. Cependant j'avais quelque chose sur le cour, c'était ma grande garde que je devais faire le lendemain.

Vendredi, 3 juillet. Lever à $6 \mathrm{hrs}$. Puis parade. A $91 / 2$ je me rends en garde pour 24 heures. L'après [midi] je me fis

38 L'auteur est devenu un vrai vétéran; il sait bien flémarder. 
rapporter par le sergent-Major parce que m'étant absenter du garde pour aller aux casernes, j'y étais demeuré trop longtemps, ce qui ne lui faisait rien du tout. Cette nuit je pense à tous mes amis que je vais revoir bientôt. Puis $3 \mathrm{hrs}$ sonnent et c'est mon tour de prendre la garde.

$\begin{array}{lrlr} & \text { hrs. A.M. } & & \text { P.M. } \\ \text { Réveillé } & 5.30 & \text { Men's Dinner } & 12.15 \\ \text { Dress Parade } & 5.45 & \text { Officers' Mess Lunch } & 1.00 \\ \text { Rations } & 6.00 & \text { Sergeants' Mess } & 1.00 \\ \text { Fall in } & 6.00 & \text { Dress for Parade } & 1.30 \\ \text { Dismiss } & 7.15 & \text { Fall in } & 2.00 \\ \text { Breakfast } & 7.30 & \text { Parade Dismiss } & 4.00 \\ \text { Sergeants' Mess } & 8.00 & \text { Souper de tous } & 6.00 \\ \text { Officers' Mess } & 8.00 & \text { Retraite } & 7.00 \\ \text { Orderly Room } & 10.00 & 1^{\text {er }} \text { poste } & 9.00 \\ \text { Dress for Parade } & 9.30 & \text { 2 poste } & 9.30 \\ \text { Dress for Guard } & 9.30 & \text { Lights out } & 10.00 \\ \text { Parade Fall in } & 10.00 & & \\ \text { Guard “ “ } & 10.00 & & \\ \text { Parade Dismiss } & 12.00 & & \end{array}$

Voici ce que nous faisons chaque jour.

Samedi, 4 juillet. Je suis de garde. Je me repose quelque peu, puis l'après-midi se passe comme d'habitude. Le soir grand pawoua. ${ }^{39} \mathrm{Au}$ lights out, l'officier du jour (Gustave Hamel) arrive et nous dit par la fenêtre d'éteindre les lumières. Il fut sifflé et dit qu'il allait rapporter le sergent Damour. Nous continuons le tapage et le Sergent Major arrivant, il fut sifflé de la plus belle manière. Alors il revient avec une lampe et nous offre de se battre. Il fut encore sifflé.

Dimanche, 5 juillet. Parade pour la messe à $9 \mathrm{hrs}$. qui eut lieu au camp. Le Père Lacombe nous fit encore ses adieux. Ils était très ému. Ensuite il eut parade à 3.30 p.m. pour se rendre aux vêpres à la mission. Le reste de la journée fut tranquille, même le soir. L'officier était un bon garçon, Capt. Dupuis. ${ }^{40}$

Lundi, 6 . Lever à $6 \mathrm{hrs.}$., puis parade jusqu'à $8 \mathrm{hrs}$. Je n'y vais pas. Après le déjeuner on apprend que les détachements de Gleichen, Crowfoot et Langdon ${ }^{41}$ avaient reçu l'ordre de reve-

39 Réunion à la manière des sauvages.

40 Lieutenant G-Vital Dupuis, $5^{\mathrm{e}}$ compagnie.

41 On pourra trouver un récit des expériences de ces détachements dans le journal de George Beauregard; voir Beauregard, Le $g^{\mathrm{me}}$ Bataillon au Nord-Ouest (Québec, 1886), 47-77. 
nir et puis continuer avec nous aux montagnes laquelle voyage devait se faire mardi à $8 \mathrm{hrs}$. du matin. Mais le soir on apprend que ce voyage ne se ferait que mercredi matin à $2 \mathrm{hrs}$. Le soir on rencontra le colonel habillé en Cow Boy. Ah la bonne farce !

Mardi, 7 juillet. Lever comme à l'ordinaire, nuais pas de parade, car il fait très mauvais. On attend le train de Gleichen à 9 hrs. A.M. Hier au soir on a fait la réception d'un nommé Rondeau ${ }^{42}$ comme franc-maçon. Il n'y avait rien de plus comique. Les officiers mêmes sont venus assister à la cérémonie et ils sont partis à $11 \mathrm{hrs}$. en disant que nous avions toujours quelque chose de nouveau pour s'amuser. Notre société porte le nom de «Aboutissement Perpetuelle ». Arrivée des détachements de Gleichen, Crowfoot et Langdon à $10 \mathrm{hrs}$. Réception de nos amis. Après le dîner, grande parade, heavy marching order, ${ }^{43}$ puis le colonel nous dit quelques mots ${ }^{44}$ et en même temps il nous dit de se préparer à partir après le souper pour aller prendre nos quartiers dans les chars, afin d'être prêts à partir au petit jour. A 91/2 P.M. départ des casernes pour les chars où nous prîmes nos quartiers. La comp. No. 7 avait un char d'immigrants avec des lits suspendus. Cependant je ne pus dormir et à $3 \mathrm{hrs}$. (mercredi le 8 juillet) nous partons de Calgarry pour Rocky Mountains. Après avoir passé 4 petites places nous arrêtons à $7 \mathrm{hrs}$. A.M. à Canmore pour prendre le déjeuner. Là nous prîmes un bon déjeuner et je visitai les alentours. D'abord j'allais voir sur un petit monticule près des montagnes qui étaient couvertes de neiges, car nous étions dans les montagnes depuis $2 \mathrm{hrs}$. Sur ce monticule il y avait 3 colonnes de granit dont les plus hautes pouvaient avoir 60 pieds de hauteur et 40 de diamètre. Je possède un petit caillou de cette colonne. Ensuite j'allai voir les engins dont un avait 10 roues très gros. Près de Canmore il y avait des pics tellement haut qu'on ne pouvait voir le bout, car les nuages passaient par dessus. Départ de Canmore à $8 \mathrm{hrs}$. Ensuite nous passons plusieurs autres petites cabanes dont chacune formait un village. A 9.05 A.M. nous arrivons à Silver City, qui est abandonné à présent, car le syndicat américain qui possédait les mines a cessé toutes recherches. Là nous changeons de méridien et

42 Arsène Rondeau, soldat, $7^{\mathrm{e}}$ compagnie.

$43 \mathrm{Par}$ exemple, on a distribué des helmets pour remplacer les scotch caps et les chapeaux de paille que les troupes portaient alors que la température était chaude.

44 D'après Beauregard (op. cit., 77) le Colonel Amyot «nous félicita de notre bonne conduite, pendant notre séjour aux différentes stations où on nous avait envoyés, et termina en nous apprenant que nous partirons demain matin pour les Montagnes Rocheuses. Le gouvernement nous procure l'agrément de ce voyage pour nous recompenser de nos loyaux services. » 
nous gagnons une heure, donc nous partons de Silver City à 8.05 A.M. A Canmore je pris une petite pierre blanche (quartz).

8 juillet, 1885. Là M. Arthur Ferland de Calgarry possède un entrepôt de provisions. A 2 milles de Laggan on entre dans la Colombie Anglaise. En effet nous entrons dans la Colombie au passage d'un petit lac. C'est alors que les plus beaux paysages s'offrent à nous, soient que nous descendions ou montions de grandes pentes; aussi nous passâmes près de hauts pics qui pouvaient avoir au moins quinze mille pieds de hauteur. ${ }^{45}$ On n'en rencontra de plus hauts, mais on ne pouvait voir la cime, les nuages les cachants. Des petits cascades descendaient de ces montagnes ou pics; puis la rivière Bow serpentait en cascades rapides faisant des bonds de 20 à 30 pds. Quelquefois le vertige nous prenait dans des endroits pendant même que la ligne allait en pente. La ligne était bordée d'un bord par un précipice de 12 à 15 pds. Puis nous passions sur des ponts de 150 à 200 pds de hauteur faits en bois etc. Le colonel veut le nous dire que nous irions jusqu'au bout du C.P.R. Nous venons d'arrêter pour ôter un gros arbre qui était tombé à travers la ligne. On passe un tunnel pratiqué sous la terre. Il était assez grand. C'est le plus considérable que nous ayons encore passé, 8 arpents. 2 autres tunnels 2 arpents et $2 \frac{1}{2}$. A 6 hrs P.M. nous arrivons dans une place d'assez belle apparence où l'on débarqua pour le souper et où nous devions passer la nuit. Le souper fut pris à l'hôtel Silkirk (sic). ${ }^{46}$ Après le souper nous allons faire une promenade à un certain pont qui traverse le "Columbia », lequel fleuve coule près des montagnes et passe à côté du village dont le nom est Donald. Il y a à peu près 1200 hommes. Notre promenade avait été en char à bras. Ensuite nous allons visiter en détail toute cette petite cité. Réellement je n'ai rien vu de si dégoutant. Il $\mathrm{y}$ a à peu près 30 filles et ce sont toutes des filles de mauvaises réputations, courant les hôtels, buvant, fumant et tirant au dés, ou jouant aux cartes avec les hommes. Une de ces filles s'est battu même avec un homme en pleine vue à propos d'argent que celui-ci lui devait. Ici je prends 2 petits cailloux dont un est un petit morceau de plombagine des montagnes. A 25 milles de Donald se trouve un pont de 300 pds de hauteur. D'ici nous sommes à 680 milles de Victoria. Le colonel nous dit que demain peut être nous irions plus loin. A $11^{1 / 2} \mathrm{hrs}$, ayant fini de visiter cette petite [ville], je reviens aux chars. Plusieurs me disent

45 Par exemple, Castle Mountain (rebaptisé Mt. Eisenhower en 1946) 9390 pieds de hauteur; Storm Mountain, 10,372 pieds; Temple Mountain, 11,636 pieds; Mt. Stephen, 10,494 pieds.

${ }^{46}$ L'Hôtel se nommait la «Selkirk House ». 
que toutes les grandes villes de Californie et des Etats-Unis ont eu un commencement semblable.

Jeudi, 9 juillet. Lever à $5 \mathrm{hrs}$. Puis nous nous rendons au même hôtel pour le déjeuner. Avant le déjeuner j'allai manger des fraises! A $61 / 2$ hrs. A.M. nous repartons, car le train ne peut nous transporter plus loin, car un feu qui dure encore a brulé les traverses et tordu les rails. Donc nous repartons pour Calgarry et de là pour Winnipeg. A $81 / 2 \mathrm{hrs} P$.M. nous arrivons à Calgarry où nous fûmes reçu par les citoyens et la bande de Calgarry. Les citoyens nous donnent le souper au Grand Central Hotel. ${ }^{47}$ Puis pour la dernière fois nous allons revoir nos casernes. A $10 \mathrm{hrs}$. nous repartons pour Winnipeg. Alors nos lettres furent distribuées, car elles étaient arrivées pendant notre voyage aux Montagnes.

Vendredi. Nous prenons le déjeuner à Maple Creek. Puis [nous] arrivons à Swift Current à $1 \mathrm{hr}$. Là il y avait un char rempli de prisonniers, 23 sauvages et métis, parmi lesquelles se trouvent Poundmaker et celui qui a tué un Père. ${ }^{48} \mathrm{Je}$ les ai vu. Ils étaient gardé par plusieurs hommes armés jusqu'aux dents. A $11 / 2 \mathrm{hrs}$ nous partons de Swift Current. En passant à Moose Jaw nous y arrêtons 2 heures pour le repas. Cet espace de temps nous donna le temps de visiter la place. C'est très beau. A 9 hrs P.M. nous arrivons à Regina. Il y avait 2 bataillons de camper; ils nous firent une belle réception. Malgré notre désir de visiter la capitale, on n'y fut qu'une demi-heure.

Samedi, 11 juillet. A 8 hrs. A.M. [nous] arrivons à Brandon où nous prenons le déjeuner à l'Edie Hotel. Après le repas, j'allai vois Pitre Lapierre de Québec dans un hôtel. Ensuite après avoir visité la ville nous embarquâmes pour Winnipeg, où on arrive

47 Le plus grand nombre des soldats ont passé les quelques heures qui leur restaient avant le départ définitif à visiter les nombreux amis qu'ils s'étaient faits pendant leur séjour à Calgary, et à faire provision de souvenirs pour leurs parents et amis de Québec. On disait que les portraits de Mgr. Grandin et du Père Lacombe disparaissaient par centaines des ateliers du photographe. Les citoyens de Calgary s'étaient rendus à la gare pour souhaiter un heureux voyage aux militaires qui depuis plusieurs mois étaient partie intégrante de la ville. Voir R. P. LeChevalier, Esquisse sur l'origine et les premiers développements de Calgary (Calgary, 1936), 72.

48 « Poundmaker et vingt-trois autres prisonniers sont détenus là. Nous avons pu les voir. Poundmaker est un grand sauvage à l'air intelligent. Il $\mathrm{y}$ a aussi un nommé Nadeau, canadien-français, que l'on me dit être l'assassin des Pères Fafard et Marchand. Tous ces prisonniers sont en route pour Régina...» (Beauregard, 87-88). Beauregard avait tort. Les Pères Fafard et Marchand ont été massacrés par les sauvages de Gros Ours au Lac-aux-grenouilles, le 2 avril 1885 ; voir R. P. LeChevalier, Batoche, (Montréal, 1941), 108-9. 
à 2 heures P.M. [Nous] prenons le déjeuner au C.P.R. Ensuite nous allons camper sur un très mauvais terrain vis-à-vis le College de Manitoba. ${ }^{49}$ Pas de nourriture. Les rations sont diminué de moitié. Ensuite il pleuvait beaucoup, tellement que nos pentalons (sic) furent encore mouillés. Le soir je sors en ville avec Alain. J'avais faim, mais pas d'argent.

Dimanche, 12 juillet. Messe au camp. La bande de St. Boniface vient avec nous à la messe. L'après-midi fut tranquille. Le soir j'allai avec Jolicœur etc. veiller chez M. Arial (Hotel de Québec) St. Boniface, où nous restâmes à une heure A.M. Retour au camp à travers les champs, perdu notre chemin. Là je rencontrai plusieurs Canadiens, d'abord un M. Côté, typographe, ancien employé de chez mon oncle Roger, puis un M. Guillaume Gauvreau, ancien ami d'Aimé Martineau.

Lundi, 13. Préparatifs pour la réception des troupes dans Winnipeg. A 9 hrs A.M. parade pour le service de Blais. ${ }^{50}$ A $91 / 2$ hrs à St. Boniface, sermon par Mgr. Tâché « Leur départ fut marqué par beaucoup de larmes et leur retour par beaucoup de joie ». Visite à la tombe de Blais et du Capt. Gagné, mort et enterré en 1870.51 Puis parade dans les rues et retour au camp. A une heure un peu de dîner; à $6 \mathrm{hrs}$ le souper; le coucher à toute heure. Nous n'avons pas de lumière dans le camp. Procession de l'armée du Salut.

Mardi, 14 juillet. Réveil à six heures. On se lève quand on le peut; déjeuner à la broche, chacun pour soi. On refuse de faire tout devoir pour cause de manqué de nourriture et de paiement. Pas de dîner ni souper, on y pense beaucoup, cependant. Le soir j'allai au Rink.

Mercredi, 15 juillet. Lever à $8 \mathrm{hrs}$. Déjeuner $10 \mathrm{hrs.} \mathrm{Exer-}$ cice à la Bayonette. Midi dîner. A $1 \mathrm{hr}$. Parade pour la réception de Middleton et Strange et du $90^{\circ}$ bataillon. Le soir j'allai encore au Rink avec Jules et J. Charles, et nous allâmes veiller chez Mde Denis... rue Princess. Retour au camp. Pendant presque toute la journée il a plu ... mouillé jusqu'aux os !

Jeudi, 16 juillet. Lever à $3 \mathrm{hrs}$. A.M. car c'était une vraie inondation. Les soldats étaient trempés, tous leurs habits mouillés. Le Col. visite toutes les tentes et prend part à nos souffran-

49 D'après Beauregard \& Les citoyens de Winnipeg nous font une belle réception. Après avoir pris le dîner au restaurant de la gare, nous partons en procession, escortés par la fanfare du Cercle Provencher de St. Boniface, et nous nous rendons jusqu'au bout de Queen Street où nous campons en face du Collège.» (p. 88).

50 Achille Blais, soldat, compagnie no 7 , décédé le 30 avril à Winnipeg. 51 A l'occasion du soulèvement des métis à la Rivière Rouge. 
ces, car lui-même avait souffert. Puis on nous permet à tous de quitter le camp pour la journée. Toute la compagnie se réunit avec le Capt. P. chez M. Sylvain, Board of Trade. Pas de nourriture au camp. Après s'être amusé jusqu'à 3 hrs P.M. on vient nous avertir que l'ordre est donnée de partir à 6 hrs P.M. Hourrahs ! Visite à la Compagnie de la Baie d'Hudson pour des souvenirs, mais tout est fermé. A 7 hrs. P.M. départ de Winnipeg. On nous applaudit. on a de mauvais chars: on ne peut dormir. Beaucoup étaient trop joyeux. Cependant tout fut tranquille.

Vendredi, 17 juillet. A $71 / 2 \mathrm{hrs}$ A.M. déjeuner à la station d'Ignace; puis nous continuons pour Port Arthur. Nous y arrivons à $31 / 2 \mathrm{hrs}$. P.M. Puis, sans nous donner le temps de prendre une bouchée, car nous n'avions pris qu'un peu de nourriture le matin, on nous entasse dans L'Alberta, vaisseau très grand et pouvant contenir beaucoup de personnes, mais non pas 1200 comme nous étions. ${ }^{52}$ Dans l'espace de 150 pieds sur 50 nous étions 600 soldats. Il est $7 \mathrm{hrs}$. Nous venons de dépasser le Port Arthur et nous entrons dans la Baie de Tonnerre, 71/4 hrs. Pas de souper ni rien, et nous étouffons, car nous sommes trop à l'étroit; à peine sommes-nous capable de s'asseoir. Et de plus il y a 12 chevaux à côté de nous, quelle peste ! Enfin après avoir attendu jusqu'à 9 hrs nous avons un magnifique souper dans le salon à laquel (sic) je fis honneur, you bet! De plus en attendant le souper je réussis à me procurer une chambre. Après le souper le Col. nous donna la permission de monter sur le pont. J'en profitai et je demeurai là à penser au retour, à ses joies, etc. A 1 heure je me couche. L'Alberta à 350 pds de longueur sur 75 .

Samedi. Lever à $8 \mathrm{hrs}$., puis déjeuner au salon. Je fais une promenade sur le deck. Le Col. me dit que nous allons être obligés de débarquer avec nos armes en passant le canal Ste Marie, ${ }^{53}$ lequel saut sépare le Lac Supérieur du Lac Huron. On est obligé de débarquer pour ne pas passer sur le territoire américain.

52 Le 9 ì̀me Bataillon avait embarqué à bord du vapeur Alberta avec le Midland Battalion de l'Ontario.

53 En 1797-98, la compagnie du Nord-Ouest construisit au Sault-SteMarie, en territoire canadien, le premier canal pour faciliter le passage de leurs canots du Lac Huron au Lac Supérieur. En 1855 le gouvernement de l'état de Michigan compléta le premier canal pour les vaisseaux. En 1870, le gouvernement des Etats-Unis refusa au Canada la permission de transporter des soldats ou des armes sur ce canal. Par conséquent, les troupes du Colonel Wolseley furent obligés de débarquer et de marcher sur le territoire canadien pour rembarquer une fois que leur vaisseau, le Chicora, eut passé le canal américain. Entre 1885 et 1895 , le gouvernement du Canada fit construire son propre canal pour les vaisseaux canadiens. 
Contre-ordre, on ne débarque, mais on est obligé de tout se mettre à fond de cale pour ne pas se faire voir des autorités américaines. A $1 \mathrm{hr}$ moins un quart, nous entrons dans le canal Ste. Marie. Après avoir attendu un temps assez, nous passons outre et nous suivons la rivière Ste. Marie qui nous conduit dans le lac Huron, et à $6 \mathrm{hrs}$. P.M. nous entrons dans le lac. Il nous semblait incroyable qu'un si gros vaisseau put passer dans une aussi petite rivière, cependant c'est le cas. Une autre chose qui nous inquiète, c'est que nous n'avons pas encore diné, et il est six heures. Enfin à $8 \mathrm{hrs}$. je réussis à dîner et souper un peu; en effet les waiters ne voulaient pas nous servir deux fois. Après avoir passé le Sault Ste. Marie, nous apercevons deux petites villes, l'une à droite Américaine et où flotte le drapeau américain, assez considérable et bien bâtie; l'autre à gauche, anglaise ou canadienne, toujours est-il que le pavillon anglais nous salua au passage. Pendant le souper, encore ici comme à Calgarry, j'entendis jouer sur le piano le morceau «Si j'étais roi » qui me rappelle tant de souvenirs. Après le souper je monte me promener sur le pont et je rève à ... coucher à $111 / 2 \mathrm{hrs}$.

Dimanche, 19 juillet. Lever à $7 \mathrm{hrs}$., déjeuner à $8 \mathrm{hrs}$. Puis nous arrivons à Owen Sound où une magnifique réception nous attendait. Les filles nous donnaient des fleurs et des bouquets, et les mettaient elles-mêmes à nos boutonnières, et nous emmenèrent un lunch magnifique. Ville assez considérable. Après le lunch toutes les jeunes filles font écrire nos noms sur des biscuits de matelots et nous demandent des souvenirs; enfin magnifique réception. Owen Sound se trouve à la Baie Georgian.

A 41/2 hrs P.M. nous partons de Owen Sound. Grande réception à Makel, ${ }^{54}$ bouquets, bande et de très jolies filles. Dundalk, réception. A Orangeville les chars passent sous une arche magnifique faite pour les troupes. A $81 / 2 \mathrm{hrs}$ P.M. nous avons un magnifique dîner à Junction de Toronto, beaucoup de dames, on joue le morceau «Si j'étais roi » pendant le souper! A $10 \mathrm{hrs}$ arrivée à Toronto. Magnifique réception, marche de 3 milles dans la ville, rues Young et King, Dublin et Reilly, bière. Départ à $11 / 2$, on presse la main des filles. On nous invite à assister à la fermeture de la Chambre qui a lieu demain.

Lundi, Lever à $7 \mathrm{hrs}$. et déjeuner à Carlton ${ }^{55}$ et nous arrivons à Ottawa à $11 \mathrm{hrs}$. A.M. Petite réception. [Nous] montons à la Chambre où nous sommes très bien reçus par les mem-

54 Markdale, Ontario.

55 Carleton Place, Ontario. 
bres. Je vais chez mon oncle Pinard et je vois beaucoup d'amis. A 6 hrs nous partons d'Ottawa, bandes et discours. A 9 hrs souper à Calumet. Nous avons assisté à la fermeture de la Chambre. A Calumet nous prenons le souper. Nous arrivons à St. Martin à $12 \mathrm{hrs.,} \mathrm{minuit...} \mathrm{difficultés} \mathrm{avec} \mathrm{le} \mathrm{conducteur} \mathrm{pour} \mathrm{le} \mathrm{chan-}$ gement des chars. Déjeuner à 3 Rivières ... .56

\section{George F. G. Stanley}

Collège Militaire Royal du Canada, Kingston, Ontario.

\section{F I N}

56 Le journal d'Arthur Potvin se termine ici. Le $9^{\text {tème }}$ bataillon est arrivé à Québec vers midi, mardi, le 21 juillet. Malgré le mauvais temps, M. Langelier, le maire de Québec, a lu une adresse de bienvenue au Colonel Amyot et à ses troupes. Escortés par les pompiers, le corps de musique du $87^{\text {lème }}$ bataillon, Batterie «A», les Zouaves, et la Garde d'Honneur de St. Sauveur, les officiers et les soldats du 9ième bataillon ont marché à l'église de St. Roch où il y eut un Te Deum chanté par l'archevêque de Québec assisté de M. Faguy, l'aumônier du régiment. Après plusieurs jours de réceptions etc., (le gouvernement mit à la disposition des troupes le vapeur Napoléon III pour une promenade sur le St. Laurent) les soldats étaient enfin libérés du service actif, lundi, le 27 juillet, à la Salle d'exercises de Québec. 